\title{
Controlled Lagrangians and the stabilization of Euler-Poincaré mechanical systems
}

\author{
Anthony M. Bloch ${ }^{1, *, \dagger}$, Naomi Ehrich Leonard ${ }^{2}$ and Jerrold E. Marsden ${ }^{3}$ \\ ${ }^{1}$ Department of Mathematics, University of Michigan, Ann Arbor, MI 48109, U.S.A. \\ ${ }^{2}$ Department of Mechanical \& Aerospace Engineering, Princeton University, Princeton, NJ 08544, U.S.A. \\ ${ }^{3}$ Control and Dynamical Systems 107-81, California Institute of Technology, Pasadena, CA 91125, U.S.A.
}

\begin{abstract}
SUMMARY
In this paper we develop a constructive approach to the determination of stabilizing control laws for a class of Lagrangian mechanical systems with symmetry — systems whose underlying dynamics are governed by the Euler-Poincaré equations. This work extends our previous work on the stabilization of mechanical control systems using the method of controlled Lagrangians. The guiding principle behind our methodology is to develop a class of stabilizing feedback control laws which yield closed-loop dynamics that remain in Lagrangian form. Using the methodology for Euler-Poincaré systems, we analyse stabilization of a satellite and an underwater vehicle controlled with momentum wheels. Copyright (C) 2001 John Wiley \& Sons, Ltd.
\end{abstract}

KEY WORDS: nonlinear control; stabilization; lagrangian systems; symmetry

\section{INTRODUCTION}

In this paper, we develop a constructive approach to the determination of stabilizing control laws for a class of Lagrangian mechanical systems with symmetry — systems described by the Euler-Poincare equations. This work complements and extends the class of systems discussed in References [1-6].

The specific case we consider here is that in which the configuration space is the Cartesian product of a non-abelian group $H$ with an Abelian group $G$ (a product of tori and lines) and where the Lagrangian is left invariant on $H$, cyclic in the Abelian variables and the controls act only on the cyclic variables.

As in our previous analysis, the guiding principle behind our methodology is to develop a class of feedback control laws whose closed-loop dynamics remain in Lagrangian, and hence

\footnotetext{
* Correspondence to: A.M. Bloch, Department of Mathematics, university of Michigan, Ann Arbor, MI48109, U.S.A.

† E-mail: abloch@math.lsa.umich.edu

Contract/grant sponsor: National Science Foundation; Contract/grant numbers: DMS-9803181, BES-9502477

Contract/grant sponsor: AFOSR; Contract/grant numbers: F49620-96-1-0100, F49620-95-1-0419

Contract/grant sponsor: Office of Naval Research; Contract/grant number: N00014-98-1-0649

Contract/grant sponsor: California Institute of Technology
}

Copyright (C) 2001 John Wiley \& Sons, Ltd. 
conservative, form and with these control laws, to achieve stabilization. We append controls that are dissipative in nature to turn the conservative stabilization into asymptotic stabilization. The dissipative case is studied in more detail in Reference [7] and related publications.

The advantage of requiring that the closed-loop dynamics be Lagrangian is that stabilization can be understood in terms of energy. In particular, we can make use of energy methods which provide a Lyapunov function and thereby give information on how to choose the control gains to achieve closed-loop stability. Further, even though work is done by the control forces, there is a modification of the mechanical energy of the system that is exactly conserved by the closed-loop dynamics; one can think of it as a combined energy available to the mechanism and the control forces. This can be used to show that, for example, for fixed control gains which achieve stabilization, the control inputs will remain bounded.

Closed-loop dynamics are guaranteed to be Lagrangian by first choosing the closed-loop Lagrangian from a class of controlled Lagrangians that we will explicitly describe. The controlled Lagrangian then provides the control law: the closed-loop dynamics are the Euler-Lagrange equations derived from the controlled Lagrangian and the new terms that appear in the dynamic equations are identified with the control forces. Specific techniques are developed for choosing the controlled Lagrangian so that new terms appear only in desired control directions. The associated theory provides sufficient conditions under which this approach will provide such a control law that yields a closed-loop system in Lagrangian form.

In this paper, we confine ourselves to controlled Lagrangians that only involve modifications to the kinetic energy of the system. One can also consider modifications to the potential energy for stabilization and tracking purposes. We have used this recently $[4,6]$ in achieving stabilization of the cart and pendulum in the full phase space and with some applications to tracking. Other relevant work involving energy methods in control and stabilization includes Krishnaprasad [8], van der Schaft [9] Aström and Furuta [10], Wang and Krishnaprasad [11], Koditschek [12], Koditschek and Rimon [13], Baillieul [14], Auckly et al. [15] and Hamberg [16].

Organization of the paper: In Section 1.1, we describe the controlled Lagrangian approach to stabilization. In Section 1.2 we describe the structure of the general class of controlled Lagrangians we consider. In Section 2, we prove a matching theorem for the case of the Euler-Poincare equations. This case is applied to the example of a spacecraft with an internal rotor in Section 3.1, and in Section 3.2 we apply the method to the problem of stabilizing an underwater vehicle using internal rotors. In Section 4 we discuss conclusions and future work.

\subsection{The controlled Lagrangian approach}

We begin by recalling, for the reader's convenience, the general controlled Lagrangian approach. Assume we have a mechanical system with an uncontrolled (free) Lagrangian equal to kinetic energy minus potential energy. We will modify the kinetic energy to produce a new controlled Lagrangian which describes the dynamics of the controlled closed-loop system.

The setting: Suppose our system has configuration space $Q$ and that a Lie group $G$ acts freely and properly on $Q$. It is useful to keep in mind the case in which $Q=S \times G$ with $G$ acting only on the second factor by group multiplication.

For example, for a rigid spacecraft with a rotor (which we treat in detail later), $Q=\mathrm{SO}(3) \times S^{1}$, where the group is $G=S^{1}$, corresponding to rotations of the rotor. 
Our goal will be to control the variables lying in the shape space $Q / G$ (in the case in which $Q=S \times G$, then $Q / G=S$ ) using controls which act directly on the variables lying in $G$. We assume that the Lagrangian is invariant under the action of $G$ on $Q$, where the action is on the factor $G$ alone. In many specific examples, such as those given below, the invariance is equivalent to the Lagrangian being cyclic in the $G$-variables. Accordingly, this produces a conservation law for the free system. Our construction, before the addition of dissipation, will preserve the invariance of the Lagrangian, thus providing us with a controlled conservation law.

Horizontal and vertical spaces: The tangent space to $Q$ can be split into a sum of horizontal and vertical parts defined as follows: for each tangent vector $v_{q}$ to $Q$ at a point $q \in Q$, we can write a unique decomposition

$$
v_{q}=\operatorname{Hor} v_{q}+\operatorname{Ver} v_{q},
$$

such that the vertical part is tangent to the orbits of the $G$-action and where the horizontal part is the metric orthogonal to the vertical space; that is, it is uniquely defined by requiring the identity

$$
g\left(v_{q}, w_{q}\right)=g\left(\operatorname{Hor} v_{q}, \text { Hor } w_{q}\right)+g\left(\operatorname{Ver} v_{q}, \operatorname{Ver} w_{q}\right)
$$

where $v_{q}$ and $w_{q}$ are arbitrary tangent vectors to $Q$ at the point $q \in Q$. This choice of horizontal space coincides with that given by the mechanical connection — see, for example, Reference [17].

The controlled Lagrangian: For the kinetic energy of our controlled Lagrangian, we use a modified version of the right-hand side of Equation (2). The potential energy remains unchanged. The modification consists of three ingredients:

(1) a different choice of horizontal space denoted Hor $_{\tau}$,

(2) a change $g \rightarrow g_{\sigma}$ of the metric acting on horizontal vectors and

(3) a change $g \rightarrow g_{\rho}$ of the metric acting on vertical vectors.

To explain these changes, we will need a little more notation." Let $\xi_{Q}$ denote the infinitesimal generator corresponding to a Lie algebra element $\xi \in \mathfrak{g}$, where $\mathfrak{g}$ is the Lie algebra of $G$. The vector field $\xi_{Q}$ may be thought of intuitively as infinitesimal group motions of the system. Thus, for each $\xi \in \mathbf{g}, \xi_{Q}$ is a vector field on the configuration manifold $Q$ and its value at a point $q \in Q$ is denoted $\xi_{Q}(q)$.

\section{Definition 1.1}

Let $\tau$ be a Lie-algebra-valued horizontal one form on $Q$; that is, a one form with values in the Lie algebra $\mathfrak{g}$ of $G$ that annihilates vertical vectors. The $\tau$-horizontal space at $q \in Q$ consists of tangent vectors to $Q$ at $q$ of the form $\operatorname{Hor}_{\tau} v_{q}=\operatorname{Hor} v_{q}-[\tau(v)]_{Q}(q)$, which also defines $v_{q} \mapsto \operatorname{Hor}_{\tau}\left(v_{q}\right)$, the $\tau$-horizontal projection. The $\tau$-vertical projection operator is defined by $\operatorname{Ver}_{\tau}\left(v_{q}\right):=\operatorname{Ver}\left(v_{q}\right)+[\tau(v)]_{Q}(q)$.

Notice that from these definitions and (1), we have

$$
v_{q}=\operatorname{Hor}_{\tau}\left(v_{q}\right)+\operatorname{Ver}_{\tau}\left(v_{q}\right)
$$

\footnotetext{
'See, for example, References [17] or [18, Chapter 9], for the relevant elementary definitions and properties of Lie groups and group actions.
} 
just as we did with $\tau$ absent. In fact, this new horizontal subspace can be regarded as defining a new connection, the $\tau$-connection. The $\tau$-horizontal space itself, which by abuse of notation, we also write as Hor $_{\tau}$ depends on $\tau$ also, but the vertical space does not - it is the tangent space to the group orbit. On the other hand, the projection map $v_{q} \mapsto \operatorname{Ver}_{\tau}\left(v_{q}\right)$ does depend on $\tau$.

\section{Definition 1.2}

Given $g_{\sigma}, g_{\rho}$ and $\tau$, we define the controlled Lagrangian to be the following Lagrangian which has the form of a modified kinetic energy minus the potential energy:

$$
L_{\tau, \sigma, \rho}(v)=\frac{1}{2}\left[g_{\sigma}\left(\operatorname{Hor}_{\tau} v_{q}, \operatorname{Hor}_{\tau} v_{q}\right)+g_{\rho}\left(\operatorname{Ver}_{\tau} v_{q}, \operatorname{Ver}_{\tau} v_{q}\right)\right]-V(q)
$$

where $V$ is the potential energy.

The equations corresponding to this Lagrangian will be our closed-loop equations. The new terms appearing in those equations corresponding to the directly controlled variables are interpreted as control inputs. The modifications to the Lagrangian are chosen so that no new terms appear in the equations corresponding to the variables that are not directly controlled. We refer to this process as 'matching'. This matching problem will be studied in detail in subsequent sections for the Euler-Poincaré class of systems of interest to us in this paper.

Another way of expressing what we are doing here is the following. A principal connection on a bundle $Q \rightarrow Q / G$, may be thought of as a Lie-algebra-valued one form and one can obtain a new connection by adding to it a horizontal one form $\tau$. The new horizontal space described in the preceding definition is exactly of this sort.

The general strategy: In outline, the general procedure that one goes through to achieve stabilization is given in the following steps.

1. Start with a mechanical system with a Lagrangian $L$ of the form kinetic minus potential energy and a symmetry group $G$.

2. Write down the equations of motion for the uncontrolled system.

3. Introduce $\tau, g_{\sigma}$ and $g_{\rho}$ to get the controlled Lagrangian (4).

4. Write down the equations of motion corresponding to the controlled Lagrangian and read off the control law $u$ from the equations in the symmetry variables (this will be a conservation law).

5. Choose $\tau, g_{\sigma}$ and $g_{\rho}$ so that the controlled Euler-Lagrange equations for the original system (i.e. the Euler-Lagrange equations for the Lagrangian $L$ with the control) agree with (that is, match) the Euler-Lagrange equations for the controlled Lagrangian $L_{\tau, \sigma, \rho}$. Determine a feedback law for $u$ by using the Euler-Lagrange equations to eliminate accelerations; then the control law becomes a feedback that is configuration and/or velocity dependent. The general matching theorem can be used to guide these calculations.

6. The stability of an equilibrium is determined by linearization or by the energy-momentum (or, when appropriate, the energy-Casimir-Arnold) method, using any available freedom in the choice of $\tau, g_{\sigma}$ and $g_{\rho}$.

7. Add dissipation to the controls to achieve asymptotic stabilization if desired.

The goal of this paper is to use this strategy to prove general matching and stabilizability theorems for Euler-Poincaré systems. The matching theorems provide sufficient conditions for successful completion of Steps 1-5 and an explicit construction of the controlled Lagrangian and 
the control law. In the case that matching is achieved, the stabilizability theorems provide sufficient conditions for closed-loop stability according to Step 6. Finally, we add dissipative control terms in our control laws to achieve asymptotic stability.

\subsection{The structure of the controlled Lagrangian}

In this section, we give a structure theorem for $L_{\tau, \sigma, \rho}$. The formula below is useful in many cases including the cases of the satellite and the underwater vehicle.

We begin by recalling the definition of the controlled Lagrangian

$$
L_{\tau, \sigma, \rho}(v)=\frac{1}{2}\left[g_{\sigma}\left(\operatorname{Hor}_{\tau} v_{q}, \operatorname{Hor}_{\tau} v_{q}\right)+g_{\rho}\left(\operatorname{Ver}_{\tau} v_{q}, \operatorname{Ver}_{\tau} v_{q}\right)\right]-V(q)
$$

and we make the following assumptions on the metric $g_{\sigma}$ :

1. $g=g_{\sigma}$ on Hor,

2. Hor and Ver are orthogonal for $g_{\sigma}$.

Keep in mind that Hor denotes the horizontal space for the given uncontrolled system and that $\mathrm{Hor}_{\tau}$ denotes the horizontal space as modified by the one form $\tau$. Note also that the new metrics $g_{\sigma}$ and $g_{\rho}$ will modify $g$ on Ver, the vertical space (or group directions), which is independent of any modification due to $\tau$. On the other hand, also recall that the vertical projection operator

$$
\operatorname{Ver}_{\tau}\left(v_{q}\right):=\operatorname{Ver}\left(v_{q}\right)+[\tau(v)]_{Q}(q)
$$

does depend on $\tau$.

Theorem 1.3

We have the following formula:

$$
L_{\tau, \sigma, \rho}(v)=L\left(v+\tau(v)_{Q}\right)+\frac{1}{2} g_{\sigma}\left(\tau(v)_{Q}, \tau(v)_{Q}\right)+\frac{1}{2} \varpi(v)
$$

where $v \in T_{q} Q$ and where

$$
\varpi(v)=\left(g_{\rho}-g\right)\left(\operatorname{Ver}_{\tau}(v), \operatorname{Ver}_{\tau}(v)\right)
$$

The proof may be found in Reference [5].

\section{EULER-POINCARÉ MATCHING AND STABILIZATION}

In this section we prove a general matching theorem in the Euler-Poincare setting, but with controls still in Abelian group directions. This will be illustrated in the next section by the spacecraft and the underwater vehicle with internal rotors.

The satellite example has two symmetry groups associated with it, as do many other examples. One group, which in this case is the non-abelian group $\mathrm{SO}(3)$, is associated with the rotational symmetry of the overall problem and another group, an Abelian group, is the product of several copies of $S^{1}$ associated to the rotors. The Abelian group is associated with the control directions, namely, the rotor variables. In this section, we use this setting to get concrete and readily implementable Euler-Poincaré matching and stabilization theorems. 


\subsection{Euler-Poincaré matching}

Motivated by these considerations, we begin with the matching problem for the case where the configuration space is the Cartesian product of a non-Abelian group $H$ with an Abelian group $G$ (which is a product of tori and lines) and where the Lagrangian is left invariant on $H$, cyclic in the Abelian variables and the controls act only on the cyclic variables.

Let $L$ denote a given left invariant Lagrangian on $T(H \times G)$. Let $l: \mathfrak{h} \times G \rightarrow \mathbb{R}$ be the restriction of $L$ to the identity of $H$ and for a curve $h(t) \in H$ let $\eta(t)=T_{h(t)} L_{h(t)^{-1}} \dot{h}$, or in abbreviated notation, $\eta(t)=h(t)^{-1} \dot{h}(t)$. Then the (reduced) Lagrangian becomes

$$
l\left(\eta^{\alpha}, \dot{\theta}^{a}\right)=\frac{1}{2} g_{\alpha \beta} \eta^{\alpha} \eta^{\beta}+g_{\alpha a} \eta^{\alpha} \dot{\theta}^{a}+\frac{1}{2} g_{a b} \dot{\theta}^{a} \dot{\theta}^{b}
$$

Here $\eta^{\alpha}$ are the variables in $\mathfrak{h}$ and $\theta^{a}$ are the control variables. Note that $g_{\alpha \beta}, g_{\alpha a}$ and $g_{a b}$ are all constant (fixed) matrices.

The conserved quantity: The conserved quantity, that is, the momentum conjugate to the cyclic variable $\theta^{a}$, is given by

$$
J_{a}=\frac{\partial l}{\partial \dot{\theta}^{a}}=g_{a \alpha} \eta^{\alpha}+g_{a b} \dot{\theta}^{b}
$$

The controlled Euler-Poincare equations: The equations of motion for the control system where the controls $u_{a}$ act in the $\theta^{a}$ directions are the controlled Euler-Poincare equations:

$$
\begin{gathered}
\frac{\mathrm{d}}{\mathrm{d} t} \frac{\partial l}{\partial \eta^{\alpha}}=c_{\alpha \delta}^{\beta} \eta^{\delta} \frac{\partial l}{\partial \eta^{\beta}} \\
\frac{\mathrm{d}}{\mathrm{d} t} \frac{\partial l}{\partial \dot{\theta}^{a}}=u_{a}
\end{gathered}
$$

where $c_{\alpha \delta}^{\beta}$ are the structure constants of the Lie algebra $\mathfrak{h}$.

Coordinate formulae for $l_{\tau, \sigma, \rho}$ : The formula from Theorem 1.3 gives in this setting

$$
\begin{aligned}
l_{\tau, \sigma, \rho}= & l\left(\eta^{\alpha}, \dot{\theta}^{a}+\tau_{\alpha}^{a} \eta^{\alpha}\right)+\frac{1}{2} \sigma_{a b} \tau_{\alpha}^{a} \tau_{\beta}^{b} \eta^{\alpha} \eta^{\beta} \\
& +\frac{1}{2} \varpi_{a b}\left(\dot{\theta}^{a}+g^{a c} g_{c \alpha} \eta^{\alpha}+\tau_{\alpha}^{a} \eta^{\alpha}\right)\left(\dot{\theta}^{b}+g^{b c} g_{c \beta} \eta^{\beta}+\tau_{\beta}^{b} \eta^{\beta}\right) .
\end{aligned}
$$

To preserve symmetry $\sigma_{a b}$ and $\rho_{a b}$ are both constant.

The controlled conserved quantity. From (11) we find that the associated controlled conserved quantity is given by

$$
\tilde{J}_{a}=\frac{\partial l_{\tau, \sigma, \rho}}{\partial \dot{\theta}^{a}}=\rho_{a b}\left(\dot{\theta}^{b}+g^{b c} g_{c \alpha} \eta^{\alpha}+\tau_{\alpha}^{b} \eta^{\alpha}\right)
$$


Euler-Poincaré equations for controlled Lagrangian: The controlled Lagrangian prescribes the closed-loop system, i.e. the closed-loop dynamics are the Euler-Poincaré equations corresponding to $l_{\tau, \sigma, \rho}$ :

$$
\begin{aligned}
\frac{\mathrm{d}}{\mathrm{d} t} \frac{\partial l_{\tau, \sigma, \rho}}{\partial \eta^{\alpha}} & =c_{\alpha \delta}^{\beta} \eta^{\delta} \frac{\partial l_{\tau, \sigma, \rho}}{\partial \eta^{\beta}} \\
\frac{\mathrm{d}}{\mathrm{d} t} \frac{\partial l_{\tau, \sigma, \rho}}{\partial \dot{\theta}^{a}} & =0
\end{aligned}
$$

To effect this closed-loop system, the control inputs $u_{a}$ must be chosen so that (13) and (14) are equivalent. Additionally, the controlled Lagrangian must satisfy matching conditions, i.e. it must be chosen so that (13) and (14) are equivalent.

Matching Euler-Poincaré expressions: To achieve matching in this case we simply need to equate

$$
\frac{\partial}{\partial \eta^{\alpha}} l \quad \text { with } \quad \frac{\partial}{\partial \eta^{\alpha}} l_{\tau, \sigma, \rho}
$$

Firstly, we have

$$
\frac{\partial}{\partial \eta^{\alpha}} l=g_{\alpha \beta} \eta^{\beta}+g_{\alpha a} \dot{\theta}^{a}
$$

Also, one gets

$$
\begin{aligned}
\frac{\partial}{\partial \eta^{\alpha}} l_{\tau, \sigma, \rho}= & \frac{\partial}{\partial \eta^{\alpha}} l\left(\eta^{\alpha}, \dot{\theta}^{a}+\tau_{\alpha}^{a} \eta^{\alpha}\right)+\frac{\partial}{\partial \dot{\theta}^{a}} l\left(\eta^{\alpha}, \dot{\theta}^{a}+\tau_{\alpha}^{a} \eta^{\alpha}\right) \tau_{\alpha}^{a} \\
& +\sigma_{a b} \tau_{\alpha}^{a} \tau_{\beta}^{b} \eta^{\beta}+\varpi_{a b}\left(\dot{\theta}^{a}+g^{a c} g_{c \alpha} \eta^{\alpha}+\tau_{\alpha}^{a} \eta^{\alpha}\right)\left(g^{b c} g_{c \alpha}+\tau_{\alpha}^{b}\right) \\
= & g_{\alpha \beta} \eta^{\beta}+g_{\alpha a}\left(\dot{\theta}^{a}+\tau_{\beta}^{a} \eta^{\beta}\right)+g_{a b} \rho^{b c} \tilde{J}_{c} \tau_{\alpha}^{a}+\sigma_{a b} \tau_{\alpha}^{a} \tau_{\beta}^{b} \eta^{\beta} \\
& +\left(\rho_{a b}-g_{a b}\right) \rho^{a d} \tilde{J}_{d}\left(g^{b c} g_{c \alpha}+\tau_{\alpha}^{b}\right)
\end{aligned}
$$

where in the second equality we used the conservation law, the definition of $\varpi$ and where the partial derivatives in this expression mean the derivatives with respect to the relevant variable slot of the function.

For matching, we need to equate (15) and (16). Since the first two terms of (16) are the same as (15) we simply need to set the remaining terms in (16) to zero. Thus, we need

$$
\left(g_{\alpha b}+\sigma_{a b} \tau_{\alpha}^{a}\right) \tau_{\beta}^{b} \eta^{\beta}+g_{a b} \rho^{b c} \tilde{J}_{c} \tau_{\alpha}^{a}+\left(\rho_{a b}-g_{a b}\right) \rho^{a d} \tilde{J}_{d}\left(g^{b c} g_{c \alpha}+\tau_{\alpha}^{b}\right)=0 .
$$

We make the following assumption:

\section{Assumption EP-1}

$$
\tau_{\alpha}^{a}=-\sigma^{a b} g_{b \alpha} .
$$

With this assumption, the first term in (17) is zero and the remaining terms are

$$
-\tilde{J}_{c} \rho^{b c}\left\{g_{a b} \sigma^{a e} g_{e \alpha}-\left(\rho_{a b}-g_{a b}\right)\left(g^{a d} g_{d \alpha}-\sigma^{a e} g_{e \alpha}\right)\right\}
$$


Thus, we require

$$
g_{a b} \sigma^{a c}-\left(\rho_{a b}-g_{a b}\right)\left(g^{a c}-\sigma^{a c}\right)=0
$$

i.e.

$$
\left(\rho_{a b}-g_{a b}\right) \rho^{b c} g^{a d}=\sigma^{c d}
$$

i.e.

$$
\sigma^{a b}+\rho^{a b}=g^{a b}
$$

Therefore, consider the following additional assumption.

Assumption EP-2

$$
\sigma^{a b}+\rho^{a b}=g^{a b} .
$$

Then, we have proved the following theorem.

\section{Theorem 2.1}

Under Assumptions EP-1 and EP-2 the Euler-Poincare equations for the controlled Lagrangian coincide with the controlled Euler-Poincaré equations.

\subsection{Determination of the control law}

The control law $u_{a}$ can be determined by comparing (10) to the controlled conservation law

$$
\frac{\mathrm{d}}{\mathrm{d} t} \frac{\partial l_{\tau, \sigma, \rho}}{\partial \dot{\theta}^{a}}=\frac{\mathrm{d}}{\mathrm{d} t} \tilde{J}_{a}=0
$$

where $\tilde{J}_{a}$ is given by (12). Since $g_{a b}$ and $\rho_{a b}$ are constant, (22) implies

$$
\frac{\mathrm{d}}{\mathrm{d} t}\left(g_{a b} \rho^{b d} \tilde{J}_{d}\right)=\frac{\mathrm{d}}{\mathrm{d} t}\left(g_{a \alpha} \eta^{\alpha}+g_{a b} \dot{\theta}^{b}+g_{a b} \tau_{\alpha}^{b} \eta^{\alpha}\right)=0
$$

Subtracting (23) from (10) gives

$$
\begin{aligned}
u_{a} & =-\frac{\mathrm{d}}{\mathrm{d} t}\left(g_{a b} \rho^{b d} \tilde{J}_{d}-J_{a}\right)=-\frac{\mathrm{d}}{\mathrm{d} t}\left(g_{a b} \tau_{\alpha}^{b} \eta^{\alpha}\right) \\
& =g_{a b} \sigma^{b c} g_{c \alpha} \dot{\eta}^{\alpha}
\end{aligned}
$$

where $J_{a}$ is given by (8) and we have used the expression in Assumption EP-1 for $\tau_{\alpha}^{b}$.

To eliminate accelerations we make use of the fact that the Euler-Poincare equations for $\eta$ hold (for both $l$ and $l_{\tau, \sigma, \rho}$ ). The Euler-Poincaré equations (9) and (10) give

$$
\begin{aligned}
\frac{\mathrm{d}}{\mathrm{d} t} \frac{\partial l}{\partial \eta^{\alpha}} & =g_{\alpha \beta} \dot{\eta}^{\beta}+g_{\alpha a} \ddot{\theta}^{a}=c_{\alpha \delta}^{\beta} \eta^{\delta} \frac{\partial l}{\partial \eta^{\beta}} \\
\frac{\mathrm{d}}{\mathrm{d} t} \frac{\partial l}{\partial \dot{\theta}^{a}} & =g_{a \alpha} \dot{\eta}^{\alpha}+g_{a b} \ddot{\theta}^{b}=u_{a}
\end{aligned}
$$

Solving (26) for $\ddot{\theta}$ gives

$$
\ddot{\theta}^{b}=g^{a b} u_{a}-g^{a b} g_{a \alpha} \dot{\eta}^{\alpha}
$$


Substituting (27) into (25), we get

$$
g_{\alpha \beta} \dot{\eta}^{\beta}+g_{\alpha b}\left(g^{a b} u_{a}-g^{a b} g_{a \beta} \dot{\eta}^{\beta}\right)=c_{\alpha \delta}^{\psi} \eta^{\delta} \frac{\partial l}{\partial \eta^{\psi}}
$$

which yields

$$
\dot{\eta}^{\beta}=B^{\alpha \beta}\left(-g_{\alpha b} g^{a b} u_{a}+c_{\alpha \delta}^{\psi} \eta^{\delta} \frac{\partial l}{\partial \eta^{\psi}}\right)
$$

where

$$
B_{\alpha \beta}=g_{\alpha \beta}-g_{\alpha b} g^{a b} g_{a \beta}
$$

Substituting (28) into (24), we get

$$
u_{d}=g_{d b} \sigma^{b c} g_{c \beta} B^{\alpha \beta}\left(-g_{\alpha e} g^{a e} u_{a}+c_{\alpha \delta}^{\psi} \eta^{\delta} \frac{\partial l}{\partial \eta^{\psi}}\right)
$$

i.e.

where

$$
u_{a}=D_{a b} \sigma^{b c} g_{c \beta} B^{\alpha \beta} c_{\alpha \delta}^{\psi} \eta^{\delta} \frac{\partial l}{\partial \eta^{\psi}}
$$

$$
D^{b a}=g^{b a}+\sigma^{b c} g_{c \beta} B^{\alpha \beta} g_{\alpha e} g^{a e}
$$

If we define control gains

$$
k_{a}^{\alpha}=D_{a b} \sigma^{b c} g_{c \beta} B^{\alpha \beta}
$$

then

$$
u_{a}=k_{a}^{\alpha} \frac{\mathrm{d}}{\mathrm{d} t} \frac{\partial l}{\partial \eta^{\alpha}}=k_{a}^{\alpha} c_{\alpha \delta}^{\psi} \eta^{\delta} \frac{\partial l}{\partial \eta^{\psi}}=k_{a}^{\alpha} c_{\alpha \delta}^{\psi} \eta^{\delta}\left(g_{\psi \beta} \eta^{\beta}+g_{\psi b} \dot{\theta}^{b}\right)
$$

\subsection{Euler-Poincaré stabilization}

Once one has the problem in Lagrangian (or Hamiltonian) form, one can proceed to use the energy-Casimir or energy-momentum method to determine stability (see e.g. References [17] or [18]). Recall that for mechanical systems, an eigenvalue analysis alone is not sufficient for determining stability.

Here we develop the general analysis of stability prior to proceeding to explicit computation in the examples. Our general approach is to assume we have a given equilibrium and a finite collection of Casimir functions (or more generally, conserved quantities) for the free Lagrangian system on the group $H$ without the introduction of the controlled variables. We then add the control variables.

To carry this out, define, using our previous notation, the (reduced) Lagrangian $l_{0}$ on $\mathfrak{h}$ by

$$
l_{0}\left(\eta^{\alpha}\right)=\frac{1}{2} g_{\alpha \beta} \eta^{\alpha} \eta^{\beta}
$$

A (relative) equilibrium $\eta_{e}$ for the corresponding dynamical equations satisfies the equation

$$
c_{\alpha \delta}^{\beta} \eta^{\delta} \frac{\partial l_{0}}{\partial \eta^{\beta}}=0
$$


Now suppose we have a collection of Casimir functions $C^{1}\left(M_{\alpha}\right), \ldots, C^{m}\left(M_{\alpha}\right)$ where

$$
M_{\alpha}=\frac{\partial l_{0}}{\partial \eta^{\alpha}}=g_{\alpha \beta} \eta^{\beta}
$$

Now set

$$
E_{\Phi}=l_{0}+\Phi\left(C^{1}, \ldots, C^{m}\right)
$$

where $\Phi$ is a smooth function.

In doing a stability analysis using the energy-Casimir method, it is standard to require that the Casimir functions be chosen so that the first variation of $E_{\Phi}$ vanishes at equilibrium, i.e.

$$
\left.\delta\left(E_{\Phi}\right)\right|_{\eta_{e}}=\left.g_{\alpha \beta} \delta \eta^{\beta}\left(\eta^{\alpha}+\sum_{k=1}^{m}\left(D_{k} \Phi\right) \frac{\partial C^{k}}{\partial M_{\alpha}}\right)\right|_{e}=0
$$

Thus, we require

$$
\left.\left(\sum_{k=1}^{m}\left(D_{k} \Phi\right) \frac{\partial C^{k}}{\partial M_{\alpha}}\right)\right|_{e}=-\eta_{e}^{\alpha}
$$

The second variation at equilibrium is given by

$$
\left.\delta^{2}\left(E_{\Phi}\right)\right|_{\eta_{e}}=\left(g_{\alpha \beta}+g_{\alpha \mu} H^{\mu v} g_{v \beta}\right) \delta \eta^{\alpha} \delta \eta^{\beta}
$$

where

$$
H^{\mu v}=\left.\left(\sum_{k, j=1}^{m}\left(D_{k j} \Phi\right) \frac{\partial C^{k}}{\partial M_{\mu}} \frac{\partial C^{j}}{\partial M_{v}}+\sum_{k=1}^{m}\left(D_{k} \Phi\right) \frac{\partial^{2} C^{k}}{\partial M_{\mu} \partial M_{v}}\right)\right|_{e}
$$

Now consider the full uncontrolled Lagrangian $l$ given by (11). Using (9), the full system will still have $\eta_{e}$ as an equilibrium together with $\dot{\theta}_{e}^{a}$ provided

$$
c_{\alpha \delta}^{\beta} \eta_{e}^{\delta}\left(g_{\beta \delta} \eta_{e}^{\delta}+g_{\beta a} \dot{\theta}_{e}^{a}\right)=0
$$

This is satisfied if $c_{\alpha \delta}^{\beta} \eta_{e}^{\delta} g_{\beta a} \dot{\theta}_{e}^{a}=0$ and in particular if $\dot{\theta}_{e}^{a}=0$. This implies, from our matching conditions, that $l_{\tau, \sigma, \rho}$ also has this equilibrium."

Set

$$
\begin{aligned}
\tilde{M}_{\alpha} & =\frac{\partial l_{\tau, \sigma, \rho}}{\partial \eta^{\alpha}}=\frac{\delta l}{\delta \eta^{\alpha}}=g_{\alpha \beta} \eta^{\beta}+g_{\alpha a} \dot{\theta}^{a} \\
& =\left(g_{\alpha \beta}-g_{a \alpha} \rho^{a b} g_{b \beta}\right) \eta^{\beta}+g_{\alpha a} \rho^{a b} \tilde{J}_{b} \\
& =G_{\alpha \beta} \eta^{\beta}+g_{\alpha a} \rho^{a b} \tilde{J}_{b}
\end{aligned}
$$

using the conserved quantities $\tilde{J}_{a}$ and where we define

$$
G_{\alpha \beta}=g_{\alpha \beta}-g_{a \alpha} \rho^{a b} g_{b \beta}
$$

\footnotetext{
"This can also be seen using the general fact that $l$ and $l_{\tau, \sigma, \rho}$ are reductions of Lagrangians $L$ and $L_{\tau, \sigma, \rho}$ that have the same 'locked Lagrangian'. This is explained in Reference [5].
} 
To examine stability of the controlled system we now use

$$
E_{\tilde{\Phi}}=l_{\tau, \sigma, \rho}+\sum_{k} \tilde{\Phi}\left(C^{k}\left(\tilde{M}_{\alpha}\right), \tilde{J}^{a}\right)
$$

where $\tilde{\Phi}$ is a smooth function. Note that $C^{k}$ are Casimir functions for the controlled system since we have only reshaped energy and not modified the Lagrangian (Hamiltonian) structure.

We next compute the first and second variations of $E_{\tilde{\Phi}}$. Using again the conserved quantities $\tilde{J}_{a}$ and assumption EP-2, we get

$$
\begin{aligned}
l_{\tau, \sigma, \rho} & =\frac{1}{2}\left(g_{\alpha \beta}+g_{a \alpha}\left(\sigma^{a b}-g^{a b}\right) g_{b \beta}\right) \eta^{\alpha} \eta^{\beta}+\frac{1}{2} \rho^{a b} \tilde{J}_{a} \tilde{J}_{b} \\
& =\frac{1}{2}\left(g_{\alpha \beta}-g_{a \alpha} \rho^{a b} g_{b \beta}\right) \eta^{\alpha} \eta^{\beta}+\frac{1}{2} \rho^{a b} \tilde{J}_{a} \tilde{J}_{b} \\
& \equiv \frac{1}{2} G_{\alpha \beta} \eta^{\alpha} \eta^{\beta}+\frac{1}{2} \rho^{a b} \tilde{J}_{a} \tilde{J}_{b}
\end{aligned}
$$

Then, we have the first variation

$$
\begin{aligned}
\delta E_{\tilde{\Phi}}= & \left.G_{\alpha \beta} \delta \eta^{\beta}\left(\eta^{\alpha}+\sum_{k=1}^{m}\left(D_{k} \tilde{\Phi}\right) \frac{\partial C^{k}}{\partial M_{\alpha}}\right)\right|_{e} \\
& +\left.\delta \tilde{J}_{a}\left(\rho^{a b} \tilde{J}_{b}+D_{m+a} \tilde{\Phi}+\sum_{k=1}^{m}\left(D_{k} \tilde{\Phi}\right) \frac{\partial C^{k}}{\partial M_{\alpha}} g_{\alpha b} \rho^{a b}\right)\right|_{e}=0
\end{aligned}
$$

Thus, in addition to condition (37), which in this context becomes

$$
\left.\left(\sum_{k=1}^{m}\left(D_{k} \tilde{\Phi}\right) \frac{\partial C^{k}}{\partial \tilde{M}_{\alpha}}\right)\right|_{e}=-\eta_{e}^{\alpha}
$$

we also require

$$
\begin{aligned}
\left.D_{m+a} \tilde{\Phi}\right|_{e} & =\left.\left(-\rho^{a b} \tilde{J}_{b}+\rho^{a b} g_{\alpha b} \eta^{\alpha}\right)\right|_{e} \\
& =\left.\left(-\rho^{a b} \tilde{J}_{b}+\left(g^{a b} g_{\alpha b}-\sigma^{a b} g_{\alpha b}\right) \eta^{\alpha}\right)\right|_{e} \\
& =-\left.\dot{\theta}^{a}\right|_{e}
\end{aligned}
$$

Similarly, we can compute the second variation of $E_{\tilde{\Phi}}$. Consider the case (apparently sufficient for applications) where

$$
\tilde{\Phi}\left(C^{1}, \ldots, C^{m}, \tilde{J}^{a}\right) \equiv \tilde{\Phi}\left(C^{1}, \ldots, C^{m}\right)+\Psi\left(\tilde{J}^{a}\right) .
$$

Accordingly, (46) becomes

$$
\left.D_{a} \Psi\right|_{e}=-\left.\dot{\theta}^{a}\right|_{e}
$$

Now define

$$
\tilde{H}^{\alpha \beta}=\left.\left(\sum_{k, j=1}^{m} D_{k j} \tilde{\Phi} \frac{\partial C^{k}}{\partial \tilde{M}_{\alpha}} \frac{\partial C^{j}}{\partial \tilde{M}_{\beta}}+\sum_{k=1}^{m} D_{k} \tilde{\Phi} \frac{\partial^{2} C^{k}}{\partial \tilde{M}_{\alpha} \partial \tilde{M}_{\beta}}\right)\right|_{e}
$$

Then, the second variation is given by

$$
\begin{aligned}
\left.\left.\delta^{2} E_{\tilde{\Phi}}\right|_{e} \equiv \delta^{2} E_{\tilde{\Phi}, \Psi}\right|_{e} & =N_{\alpha \beta} \delta \eta^{\alpha} \delta \eta^{\beta}+2 P_{\alpha}^{a} \delta \eta^{\alpha} \delta \tilde{J}_{a}+R^{a b} \delta \tilde{J}_{a} \delta \tilde{J}_{b} \\
& =N_{\alpha \beta} \delta \xi^{\alpha} \delta \xi^{\beta}+\left(R^{a b}-N^{\gamma \delta} P_{\gamma}^{a} P_{\delta}^{b}\right) \delta \tilde{J}_{a} \delta \tilde{J}_{b}
\end{aligned}
$$


where

$$
\begin{gathered}
N_{\alpha \beta}=G_{\alpha \beta}+G_{\alpha \gamma} \tilde{H}^{\gamma \delta} G_{\delta \beta} \\
P_{\alpha}^{a}=G_{\alpha \gamma} \tilde{H}^{\gamma \delta} g_{\delta b} \rho^{a b} \\
R^{a b}=\rho^{a b}+\left.\left(\frac{\partial^{2} \Psi}{\partial \tilde{J}_{a} \partial \tilde{J}_{b}}\right)\right|_{e}+\tilde{H}^{\alpha \beta} g_{\alpha c} \rho^{a c} g_{\beta d} \rho^{b d}
\end{gathered}
$$

and

$$
\delta \xi^{\alpha}=\delta \eta^{\alpha}+N^{\alpha \beta} P_{\beta}^{b} \delta \tilde{J}_{b}
$$

Definiteness of this quantity at the given equilibrium yields nonlinear stability. Using the freedom in choosing $\left.\left(\partial^{2} \Psi / \partial \tilde{J}_{a} \partial \widetilde{J}_{b}\right)\right|_{e}$ we can make the second term on the right-hand side of (50) have whatever definiteness we require. Then, stability will be guaranteed if we can choose $\rho_{a b}$ such that $N_{\alpha \beta}$ is definite (under the restrictions that (45) and (46) are satisfied). We summarize these findings in the following result.

Theorem 2.2.

Let $\eta_{e}$ be an equilibrium for the uncontrolled dynamics given by $l_{0}$ (33). Suppose that $\dot{\theta}_{e}$ satisfies (40). Then, $\left(\eta_{e}, \dot{\theta}_{e}\right)$ is an equilibrium for the controlled system described by $l_{\tau, \sigma, \rho}(43)$. This equilibrium is Lyapunov stable for the controlled dynamics if $\rho_{a b}$ and $\tilde{\Phi}\left(C_{1}, \ldots, C_{m}\right)$ can be found so that (45) is satisfied and $G_{\alpha \beta}+G_{\alpha \gamma} \tilde{H}^{\gamma \delta} G_{\delta \beta}$ is definite at the equilibrium.

Recall that if the equilibrium is spectrally unstable for the uncontrolled dynamics, it won't be possible to make $g_{\alpha \beta}+g_{\alpha \gamma} H^{\gamma \delta} g_{\delta \beta}$ definite. In the controlled setting, however, we modify the metric so that now it can be possible to make $G_{\alpha \beta}+G_{\alpha \gamma} \tilde{H}^{\gamma \delta} G_{\delta \beta}$ definite. The matrix $G_{\alpha \beta}$ can be interpreted as the horizontal part of the metric for the controlled system, i.e., the "controlled inertia' associated with the group $H$ variables. Since $G_{\alpha \beta}=g_{\alpha \beta}-g_{a \alpha} \rho^{a b} g_{b \beta}$, it is clear how the control gain $\rho_{a b}$ enters in to provide stabilization, i.e. by modifying the inertia.

To obtain asymptotic stability, we introduce an additional term in the control law to simulate dissipation. Let the complete control law be

$$
u_{a}=u_{a}^{\text {cons }}+g_{a b} \rho^{b c} u_{c}^{\text {diss }}
$$

where

$$
u_{a}^{\text {cons }}=g_{a b} \sigma^{b c} g_{c \alpha} \dot{\eta}^{\alpha}
$$

i.e. the control law term derived above in equation (24), and where $u_{a}^{\text {diss }}$ is the new feedback term that will simulate dissipation. With this control law, the controlled dynamics are computed to be

$$
\begin{aligned}
& \frac{\mathrm{d}}{\mathrm{d} t} \frac{\partial l_{\tau, \sigma, \rho}}{\partial \eta^{\alpha}}=c_{\alpha \delta}^{\beta} \eta^{\delta} \frac{\partial l_{\tau, \sigma, \rho}}{\partial \eta^{\beta}} \\
& \frac{\mathrm{d}}{\mathrm{d} t} \frac{\partial l_{\tau, \sigma, \rho}}{\partial \dot{\theta}^{a}}=\dot{\tilde{J}}_{a}=u_{a}^{\mathrm{diss}}
\end{aligned}
$$

We choose $u_{a}^{\text {diss }}$ so that $(\mathrm{d} / \mathrm{d} t) E_{\tilde{\Phi}}$ is non-decreasing (non-increasing) if the equilibrium is a local maximum (minimum) of $E_{\tilde{\Phi}}$. We then carry out a LaSalle invariance principle analysis (details can be found in References [7]). 
Assume that $\tilde{\Phi}$ can be taken in form (47). Then

$$
\begin{aligned}
\frac{\mathrm{d}}{\mathrm{d} t} E_{\tilde{\Phi}} & =\frac{\mathrm{d}}{\mathrm{d} t} l_{\tau, \sigma, \rho}+\frac{\partial \tilde{\Phi}}{\partial C^{k}} \dot{C}^{k}+\frac{\partial \Psi}{\partial \tilde{J}_{a}} \dot{\tilde{J}}_{a} \\
& =\dot{\theta}^{a} u_{a}^{\mathrm{diss}}+\frac{\partial \Psi}{\partial \tilde{J}_{a}} u_{a}^{\mathrm{diss}} \\
& =\left(\dot{\theta}^{a}+\frac{\partial \Psi}{\partial \tilde{J}_{a}}\right) u_{a}^{\mathrm{diss}}
\end{aligned}
$$

where we have used the fact that $(\mathrm{d} / \mathrm{d} t) l_{\tau, \sigma, \rho}=\dot{\theta}^{a} u_{a}^{\mathrm{diss}}$. We also used the fact that

$$
C^{k}=\text { constant }
$$

since the actuation is internal.

Without loss of generality, we may assume that $E_{\tilde{\Phi}}$ has a local maximum at the equilibrium. Choose

where $c_{a b}$ is a positive-definite matrix. Then,

$$
u_{a}^{\mathrm{diss}}=c_{a b}\left(\dot{\theta}^{b}+\frac{\partial \Psi}{\partial \widetilde{J}_{b}}\right)
$$

$$
\frac{\mathrm{d}}{\mathrm{d} t} E_{\tilde{\Phi}}=c_{a b}\left(\dot{\theta}^{a}+\frac{\partial \Psi}{\partial \tilde{J}_{a}}\right)\left(\dot{\theta}^{b}+\frac{\partial \Psi}{\partial \tilde{J}_{b}}\right) \geqslant 0
$$

In the case that the equilibrium of interest is such that $\left.\dot{\theta}^{a}\right|_{e}=0$, we can take $\Psi$ as

$$
\Psi(\tilde{J})=\frac{1}{2} \varepsilon^{b c} \tilde{J}_{b} \tilde{J}_{c}
$$

where $\varepsilon^{b c}$ is a sign definite symmetric matrix. Then, (59) becomes

$$
\frac{\mathrm{d}}{\mathrm{d} t} E_{\tilde{\Phi}}=c_{a b}\left(\dot{\theta}^{a}+\varepsilon^{a c} \tilde{J}_{c}\right)\left(\dot{\theta}^{b}+\varepsilon^{b d} \tilde{J}_{d}\right) \geqslant 0
$$

To obtain asymptotic stability we use the LaSalle invariance principle and the details of the specific system. In Reference [7] the general theory of how to carry this out is examined in some detail. See also Woolsey and Leonard [19].

\section{GYROSCOPIC STABILIZATION WITH ROTORS}

In this section we show how the preceding results on Euler-Poincare matching and stabilization apply to an important class of examples, namely rigid bodies carrying internal rotors. We treat both the spacecraft with internal rotors and the underwater vehicle with internal rotors.

\subsection{The rigid spacecraft with a rotor}

Following Krishnaprasad [8] and Bloch et al. [20], we consider a rigid body with a rotor aligned along the third principal axis of the body as in Figure 1. The rotor spins under the influence of a torque $u$ acting on the rotor. The configuration space is $Q=S O(3) \times S^{1}$, with the first factor $H=S O(3)$ being the spacecraft attitude and the second factor $G=S^{1}$ being the rotor angle. The 
Lagrangian is total kinetic energy of the system, (rigid carrier plus rotor), with no potential energy.

The Lagrangian: The reduced Lagrangian on $\mathfrak{s} \mathbf{v}(3) \times S^{1}$ for this system is

$$
\begin{gathered}
l(\Omega, \dot{\phi})=\frac{1}{2}\left(\lambda_{1} \Omega_{1}^{2}+\lambda_{2} \Omega_{2}^{2}+I_{3} \Omega_{3}^{2}+J_{3}\left(\Omega_{3}+\dot{\phi}\right)^{2}\right) \\
=\frac{1}{2}\left[\begin{array}{llll}
\Omega_{1} & \Omega_{2} & \Omega_{3} & \dot{\phi}
\end{array}\right]\left[\begin{array}{cccc}
\lambda_{1} & 0 & 0 & 0 \\
0 & \lambda_{2} & 0 & 0 \\
0 & 0 & I_{3}+J_{3} & J_{3} \\
0 & 0 & J_{3} & J_{3}
\end{array}\right]\left[\begin{array}{c}
\Omega_{1} \\
\Omega_{2} \\
\Omega_{3} \\
\dot{\phi}
\end{array}\right]
\end{gathered}
$$

where $\Omega=\left(\Omega_{1}, \Omega_{2}, \Omega_{3}\right)$ is the body angular velocity vector of the carrier, $\phi$ is the relative angle of the rotor, $I_{1}>I_{2}>I_{3}$ are the rigid body moments of inertia, $J_{1}=J_{2}$ and $J_{3}$ are the rotor moments of inertia and $\lambda_{i}=I_{i}+J_{i}$.

The body angular momenta are determined by the Legendre transform to be

$$
\begin{aligned}
& \Pi_{1}=\lambda_{1} \Omega_{1} \\
& \Pi_{2}=\lambda_{2} \Omega_{2} \\
& \Pi_{3}=\lambda_{3} \Omega_{3}+J_{3} \dot{\phi}
\end{aligned}
$$

Note that $\Pi_{\alpha}=\tilde{M}_{\alpha}$ following the notation of (41). The momentum conjugate to $\phi$ is

$$
\frac{\partial l}{\partial \dot{\phi}}=l_{3}=J_{3}\left(\Omega_{3}+\dot{\phi}\right)
$$

Equations of motion: The equations of motion with a control torque $u$ acting on the rotor are

$$
\begin{aligned}
\lambda_{1} \dot{\Omega}_{1} & =\lambda_{2} \Omega_{2} \Omega_{3}-\left(\lambda_{3} \Omega_{3}+J_{3} \dot{\phi}\right) \Omega_{2} \\
\lambda_{2} \dot{\Omega}_{2} & =-\lambda_{1} \Omega_{1} \Omega_{3}+\left(\lambda_{3} \Omega_{3}+J_{3} \dot{\phi}\right) \Omega_{1} \\
\lambda_{3} \dot{\Omega}_{3}+J_{3} \ddot{\phi} & =\left(\lambda_{1}-\lambda_{2}\right) \Omega_{1} \Omega_{2} \\
\dot{l}_{3} & =u
\end{aligned}
$$

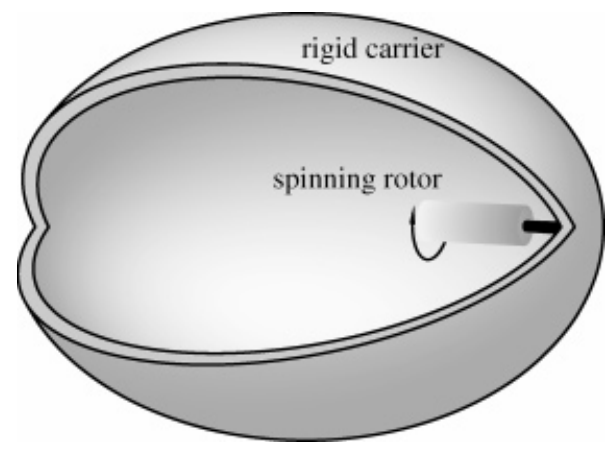

Figure 1. The spacecraft with the rotor attached along the long axis. 
Controlled Lagrangian and matching: Next, we form the controlled Lagrangian and apply the Euler-Poincare matching theorem. Since the Abelian group $G=S^{1}$ is one-dimensional, $g_{a b}$, $\sigma_{a b}$ and $\rho_{a b}$ are all scalars. From (61), $g_{a b}=J_{3}$. We let $\sigma_{a b}=\sigma J_{3}$ and $\rho_{a b}=\rho J_{3}$ where $\sigma$ and $\rho$ are dimensionless scalars. For matching we should choose $\tau_{\alpha}^{a}$ according to Assumption EP-1, i.e.

$$
\left(\begin{array}{lll}
\tau_{\Omega_{1}}^{\phi} & \tau_{\Omega_{2}}^{\phi} & \tau_{\Omega_{3}}^{\phi}
\end{array}\right)=-\frac{1}{\sigma J_{3}}\left(\begin{array}{lll}
0 & 0 & J_{3}
\end{array}\right)
$$

Further, in order to satisfy Assumption EP-2 $\rho$ should satisfy

$$
\frac{1}{\sigma J_{3}}+\frac{1}{\rho J_{3}}=\frac{1}{J_{3}}
$$

which implies

$$
\rho=\frac{\sigma}{\sigma-1}
$$

Substituting into Equation (11) with these choices, the controlled Lagrangian is given by

$$
\begin{aligned}
l_{\tau, \sigma, \rho} & =\frac{1}{2}\left(\lambda_{1} \Omega_{1}^{2}+\lambda_{2} \Omega_{2}^{2}+I_{3} \Omega_{3}^{2}+\frac{1}{\sigma} J_{3} \Omega_{3}^{2}+\rho J_{3}\left(\Omega_{3}+\dot{\phi}-\frac{1}{\sigma} \Omega_{3}\right)^{2}\right) \\
& =\frac{1}{2}\left(\lambda_{1} \Omega_{1}^{2}+\lambda_{2} \Omega_{2}^{2}+I_{3} \Omega_{3}^{2}+\frac{1}{\sigma} J_{3} \Omega_{3}^{2}+\frac{\sigma}{\sigma-1} J_{3}\left(\Omega_{3}+\dot{\phi}-\frac{1}{\sigma} \Omega_{3}\right)^{2}\right)
\end{aligned}
$$

where $\sigma$ is a free variable and matching is ensured by Theorem 2.1. The controlled conserved quantity is

$$
\tilde{l}_{3}=\frac{\partial l_{\tau, \sigma, \rho}}{\partial \dot{\phi}}=J_{3} \Omega_{3}+\rho J_{3} \dot{\phi}
$$

Control law: Using (24), the control law is

$$
u=\frac{1}{\sigma} J_{3} \dot{\Omega}_{3}
$$

To get the control law with accelerations eliminated we use formula (32). We compute from (29) the matrix $B$ to be diagonal with diagonal elements $\left(\lambda_{1}, \lambda_{2}, I_{3}\right)$. We then compute $D$ from (30) to be

$$
D^{\phi \phi}=\frac{1}{J_{3}}+\frac{1}{\sigma} \frac{1}{I_{3}}
$$

Using (31), we find the control gains $k_{a}^{\alpha}$

$$
\left(\begin{array}{lll}
k_{\phi}^{\Omega_{1}} & k_{\phi}^{\Omega_{2}} & k_{\phi}^{\Omega_{3}}
\end{array}\right)=\left(\begin{array}{lll}
0 & 0 & \frac{J_{3}}{\sigma I_{3}+J_{3}}
\end{array}\right)
$$

Letting $k=k_{\phi}^{\Omega_{3}}$ and using (32), the control law is

$$
u=k\left(\lambda_{1}-\lambda_{2}\right) \Omega_{1} \Omega_{2}
$$


We note that

$$
\frac{1}{\sigma}=\frac{k}{1-k} \frac{I_{3}}{J_{3}}
$$

Stabilization: As in Reference [20], we consider the equilibrium $\left(\Omega_{1}, \Omega_{2}, \Omega_{3}, \dot{\phi}\right)=(0, \bar{\Omega}, 0,0)$ corresponding to steady rotation about the intermediate axis (unstable for the uncontrolled spacecraft). In contrast to our earlier work, however, we carry out our analysis on the Lagrangian side and we do not restrict the stability analysis to the zero level set of the conserved momentum.

Casimir functions for this problem are functions of the total angular momentum of the body plus rotor system. We let

$$
\begin{aligned}
C & =\frac{1}{2}\left(\Pi_{1}^{2}+\Pi_{2}^{2}+\Pi_{3}^{2}\right) \\
& =\frac{1}{2}\left(\left(\lambda_{1} \Omega_{1}\right)^{2}+\left(\lambda_{2} \Omega_{2}\right)^{2}+\left(\left(I_{3}+\frac{J_{3}}{\sigma}\right) \Omega_{3}+\frac{\sigma-1}{\sigma} \tilde{l}_{3}\right)^{2}\right)
\end{aligned}
$$

Thus, our Lyapunov function for studying stability becomes

$$
E_{\tilde{\Phi}, \Psi}=\frac{1}{2}\left(\lambda_{1} \Omega_{1}^{2}+\lambda_{2} \Omega_{2}^{2}+\left(I_{3}+\frac{J_{3}}{\sigma}\right) \Omega_{3}^{2}+\frac{1}{\rho J_{3}} \tilde{l}_{3}^{2}\right)+\tilde{\Phi}(C)+\Psi\left(\tilde{l}_{3}\right)
$$

To satisfy (45) for Theorem 2.2, the first derivative of $\tilde{\Phi}$ evaluated at the equilibrium $\left.\tilde{\Phi}^{\prime}\right|_{e}$ should satisfy

$$
\left.\tilde{\Phi}^{\prime}\right|_{e} \lambda_{2} \bar{\Omega}_{2}=-\bar{\Omega}_{2}
$$

i.e.

$$
\left.\tilde{\Phi}^{\prime}\right|_{e}=-\frac{1}{\lambda_{2}}
$$

Given this criterion, we need only show that we can make $N_{\alpha \beta}=G_{\alpha \beta}+G_{\alpha \gamma} \tilde{H}^{\gamma \delta} G_{\delta \beta}$ definite. In this case we compute the matrix with elements $G_{\alpha \beta}=g_{\alpha \beta}-g_{a \alpha} \rho^{a b} g_{b \beta}$ to be the diagonal matrix $\operatorname{diag}\left(\lambda_{1}, \lambda_{2}, I_{3}+J_{3} / \sigma\right)$. The matrix with elements $\tilde{H}^{\alpha \beta}$ as defined by (49) is computed to be

$$
\begin{aligned}
\tilde{H} & =\operatorname{diag}\left(\left.\tilde{\Phi}^{\prime}\right|_{e},\left.\tilde{\Phi}^{\prime}\right|_{e}+\left.\tilde{\Phi}^{\prime \prime}\right|_{e} \lambda_{2}^{2} \bar{\Omega}^{2},\left.\tilde{\Phi}^{\prime}\right|_{e}\right) \\
& =\operatorname{diag}\left(-\frac{1}{\lambda_{2}},-\frac{1}{\lambda_{2}}+\left.\tilde{\Phi}^{\prime \prime}\right|_{e} \lambda_{2}^{2} \bar{\Omega}^{2},-\frac{1}{\lambda_{2}}\right)
\end{aligned}
$$

Using this, we compute the matrix with elements $N_{\alpha \beta}$ to be

$$
N=\operatorname{diag}\left(\lambda_{1}-\frac{\lambda_{1}^{2}}{\lambda_{2}},\left.\tilde{\Phi}^{\prime \prime}\right|_{e} \lambda_{2}^{4} \bar{\Omega}^{2}, I_{3}+\frac{J_{3}}{\sigma}-\frac{1}{\lambda_{2}}\left(I_{3}+\frac{J_{3}}{\sigma}\right)^{2}\right)
$$

Since the first diagonal element is negative, we choose $\left.\tilde{\Phi}^{\prime \prime}\right|_{e}$ to be negative also and require that

$$
I_{3}+\frac{J_{3}}{\sigma}-\frac{1}{\lambda_{2}}\left(I_{3}+\frac{J_{3}}{\sigma}\right)^{2}<0
$$

This condition holds if $k>1-I_{3} / \lambda_{2}$. Therefore, by Theorem 2.2 we have proved. 


\section{Proposition 3.1}

For $k>1-I_{3} / \lambda_{2}$, the equilibrium $(0, \bar{\Omega}, 0,0)$ is nonlinearly stable for the feedback controlled system where $u$ is given by (68).

The stabilization that takes place as the gain is increased can be viewed in terms of a modification of the phase portrait of the rigid body: the four heteroclinic orbits for the rigid body close up along the 'hinge' joining the two saddle points forming a circle of fixed points and then open up along a 'hinge' joining two stable points, forming a stability island where there were saddle points previously.

One of the advantages of the approach in this paper is that it is systematic, given the class of control Lagrangians we have proposed (of course, we are not excluding the possibility that other interesting classes might be found). For example, with the satellite with rotors one can readily deal with variants of the problem such as putting the rotor along the short axis instead of the long one, or with its axis in some direction other than a principal axis direction.

Asymptotic stability: We now add further control to the system to achieve asymptotic stability of the equilibrium. As in Section 2 we choose

$$
\Psi\left(\tilde{l}_{3}\right)=\frac{1}{2 \varepsilon J_{3}} \tilde{l}_{3}^{2}
$$

with $\varepsilon<0$ and $|\varepsilon| \ll 1$. By (58) we choose a control of the form

$$
u^{\mathrm{diss}}=c\left(\dot{\phi}+\frac{1}{\varepsilon J_{3}} \tilde{l}_{3}\right)=c\left(\frac{1}{\varepsilon} \Omega_{3}+\left(1+\frac{\rho}{\varepsilon}\right) \dot{\phi}\right)
$$

with $c>0$. As is shown in Reference [7] this leads to asymptotic stability.

\subsection{The dynamics of an underwater vehicle}

The dynamics of an underwater vehicle provides another rich example of the methods of the present paper. The techniques proceed somewhat similarly to those for the satellite to show what explicit gains are needed to stabilize an otherwise unstable motion. The underwater vehicle example is much richer, however, because it interacts with the surrounding fluid for both rotational and translational motions, whereas the satellite stabilization problem deals only with the rotational dynamics. Some of the relative equilibria that are of interest are discussed in References [21, 22]. Stabilization of the underwater vehicle with internal rotors was first investigated in Reference [23].

The dynamical model: We model the underwater vehicle using Kirchhoff's equations which describe the dynamics of a rigid body in an ideal, unbounded fluid. We assume that the vehicle is neutrally buoyant (buoyant force equal and opposite to gravitational force) and for simplicity, we assume that the vehicle has three planes of symmetry and uniformly distributed mass. The latter assumption implies that the centre of gravity and centre of buoyancy are coincident. In reality, the centre of gravity is designed to be lower than the centre of buoyancy for stability. By making the centres coincident, the stability problem becomes more demanding. Our simplifying assumptions also imply that the matrix of mass, inertia, added mass and added inertia of the body-fluid system can be diagonalized (for further details see, for example, Reference [21]). We will denote the diagonal elements of the mass plus added mass matrix by $\left(m_{1}, m_{2}, m_{3}\right)$ and the diagonal elements of the inertia plus added inertia matrix by $\left(I_{1}, I_{2}, I_{3}\right)$. 
We consider the underwater vehicle with two independently controlled, symmetric, internal rotors, one aligned along the first principal axis and the other aligned along the second principal axis. The first rotor spins under the influence of a torque $u_{1}$ acting on it, and the second rotor spins under the influence of a torque $u_{2}$ acting on it.

The configuration space of the underwater vehicle plus rotors system is $Q=S E(3) \times\left(S^{1} \times S^{1}\right)$ with the first factor $H=S E(3)$ being the underwater vehicle attitude and position and the second factor $G=S^{1} \times S^{1}$ being the pair of rotor angles. The Lagrangian is the total kinetic energy of the system with no potential energy.

The Lagrangian: The reduced Lagrangian on $\mathfrak{s e}(3) \times\left(S^{1} \times S^{1}\right)$ for this system is

$$
\begin{aligned}
& l\left(v, \Omega, \dot{\alpha}_{1}, \dot{\alpha}_{2}\right) \\
& =\frac{1}{2}\left(m_{1} v_{1}^{2}+m_{2} v_{2}^{2}+m_{3} v_{3}^{2}+\bar{I}_{1} \Omega_{1}^{2}+\bar{I}_{2} \Omega_{2}^{2}+\lambda_{3} \Omega_{3}^{2}+J_{1}\left(\Omega_{1}+\dot{\alpha}_{1}\right)^{2}+J_{2}\left(\Omega_{2}+\dot{\alpha}_{2}\right)^{2}\right) \\
& =\frac{1}{2}\left[\begin{array}{c}
v_{1} \\
v_{2} \\
v_{3} \\
\Omega_{1} \\
\Omega_{2} \\
\Omega_{3} \\
\dot{\alpha}_{1} \\
\dot{\alpha}_{2}
\end{array}\right]\left[\begin{array}{cccccccc}
m_{1} & 0 & 0 & 0 & 0 & 0 & 0 & 0 \\
0 & m_{2} & 0 & 0 & 0 & 0 & 0 & 0 \\
0 & 0 & m_{3} & 0 & 0 & 0 & 0 & 0 \\
0 & 0 & 0 & \lambda_{1} & 0 & 0 & J_{1} & 0 \\
0 & 0 & 0 & 0 & \lambda_{2} & 0 & 0 & J_{2} \\
0 & 0 & 0 & 0 & 0 & \lambda_{3} & 0 & 0 \\
0 & 0 & 0 & J_{1} & 0 & 0 & J_{1} & 0 \\
0 & 0 & 0 & 0 & J_{2} & 0 & 0 & J_{2}
\end{array}\right]\left[\begin{array}{l}
v_{1} \\
v_{2} \\
v_{3} \\
\Omega_{1} \\
\Omega_{2} \\
\Omega_{3} \\
\dot{\alpha}_{1} \\
\dot{\alpha}_{2}
\end{array}\right]
\end{aligned}
$$

where $v=\left(v_{1}, v_{2}, v_{3}\right)$ is the body linear velocity of the vehicle, $\Omega=\left(\Omega_{1}, \Omega_{2}, \Omega_{3}\right)$ is the body angular velocity vector of the vehicle, $\alpha_{i}$ is the relative angle of the $i$ th rotor, $i=1,2, J_{1}$ and $J_{2}^{1}=J_{3}^{1}$ are the first rotor moments of inertia, $J_{2}$ and $J_{1}^{2}=J_{3}^{2}$ are the second rotor moments of inertia, $\bar{I}_{1}=I_{1}+J_{1}^{2}, \bar{I}_{2}=I_{2}+J_{2}^{1}, \lambda_{3}=\bar{I}_{3}=I_{3}+J_{3}^{1}+J_{3}^{2}$ and $\lambda_{i}=\bar{I}_{i}+J_{i}$, $i=1,2$.

The vehicle linear and angular momenta are determined by the Legendre transform to be

$$
\begin{aligned}
& P_{1}=m_{1} v_{1} \\
& P_{2}=m_{2} v_{2} \\
& P_{3}=m_{3} v_{3} \\
& \Pi_{1}=\lambda_{1} \Omega_{1}+J_{1} \dot{\alpha}_{1} \\
& \Pi_{2}=\lambda_{2} \Omega_{2}+J_{2} \dot{\alpha}_{2} \\
& \Pi_{3}=\lambda_{3} \Omega_{3}
\end{aligned}
$$

Note that $\tilde{M}_{i}=P_{i}, i=1,2,3$ and $\tilde{M}_{i+3}=\Pi_{i}, i=1,2,3$ following the notation of (41). The momenta conjugate to $\alpha_{1}$ and $\alpha_{2}$ are

$$
\begin{gathered}
\frac{\partial l}{\partial \dot{\alpha}_{1}}=l_{1}=J_{1}\left(\Omega_{1}+\dot{\alpha}_{1}\right) \\
\frac{\partial l}{\partial \dot{\alpha}_{2}}=l_{2}=J_{2}\left(\Omega_{2}+\dot{\alpha}_{2}\right)
\end{gathered}
$$


Equations of motion: The equations of motion with the control torques $u_{1}$ and $u_{2}$ acting on the rotors are

$$
\begin{aligned}
\dot{\Pi} & =\Pi \times \Omega+P \times v \\
\dot{P} & =P \times \Omega \\
\dot{l}_{1} & =u_{1} \\
\dot{l}_{2} & =u_{2}
\end{aligned}
$$

Controlled Lagrangian and matching: To develop our controller, we next form the controlled Lagrangian and apply the Euler-Poincaré matching theorem. From (71), $g_{a b}$ is given by

$$
\left[\begin{array}{ll}
g_{\alpha_{1} \alpha_{1}} & g_{\alpha_{1} \alpha_{2}} \\
g_{\alpha_{2} \alpha_{1}} & g_{\alpha_{2} \alpha_{2}}
\end{array}\right]=\left[\begin{array}{cc}
J_{1} & 0 \\
0 & J_{2}
\end{array}\right]
$$

Let $\sigma_{1}, \sigma_{2}$ be dimensionless scalars and let $\sigma_{a b}$ be given by

$$
\left[\begin{array}{cc}
\sigma_{\alpha_{1} \alpha_{1}} & \sigma_{\alpha_{1} \alpha_{2}} \\
\sigma_{\alpha_{2} \alpha_{1}} & \sigma_{\alpha_{2} \alpha_{2}}
\end{array}\right]=\left[\begin{array}{cc}
J_{1} \sigma_{1} & 0 \\
0 & J_{2} \sigma_{2}
\end{array}\right]
$$

Similarly, let $\rho_{1}, \rho_{2}$ be dimensionless scalars and let $\rho_{a b}$ be given by

$$
\left[\begin{array}{cc}
\rho_{\alpha_{1} \alpha_{2}} & \rho_{\alpha_{1} \alpha_{2}} \\
\rho_{\alpha_{2} \alpha_{2}} & \rho_{\alpha_{2} \alpha_{2}}
\end{array}\right]=\left[\begin{array}{cc}
J_{1} \rho_{1} & 0 \\
0 & J_{2} \rho_{2}
\end{array}\right]
$$

Note that we did not have to choose zero off-diagonal terms for the above. We have chosen to use a diagonal gain matrix for simplicity, but matching does allow a more general gain matrix. For matching we should choose $\tau_{\alpha}^{a}$ according to Assumption EP-1, i.e.

$$
\left[\begin{array}{llllll}
\tau_{v_{1}}^{\alpha_{1}} & \tau_{v_{2}}^{\alpha_{1}} & \tau_{v_{2}}^{\alpha_{1}} & \tau_{\Omega_{1}}^{\alpha_{1}} & \tau_{\Omega_{2}}^{\alpha_{1}} & \tau_{\Omega_{3}}^{\alpha_{1}} \\
\tau_{v_{1}}^{\alpha_{2}} & \tau_{v_{2}}^{\alpha_{2}} & \tau_{v_{2}}^{\alpha_{2}} & \tau_{\Omega_{1}}^{\alpha_{2}} & \tau_{\Omega_{2}}^{\alpha_{2}} & \tau_{\Omega_{3}}^{\alpha_{2}}
\end{array}\right]=\left[\begin{array}{cccccc}
0 & 0 & 0 & \frac{1}{\sigma_{1}} & 0 & 0 \\
0 & 0 & 0 & 0 & \frac{1}{\sigma_{2}} & 0
\end{array}\right]
$$

Further, by assumption EP-2, $\rho$ should satisfy for $i=1,2$,

$$
\frac{1}{\sigma_{i} J_{i}}+\frac{1}{\rho_{i} J_{i}}=\frac{1}{J_{i}}
$$

which implies

$$
\rho_{i}=\frac{\sigma_{i}}{\sigma_{i}-1}
$$

Plugging into (2.5) with these choices, the controlled Lagrangian is given by

$$
\begin{aligned}
l_{\tau, \sigma, \rho}(v, \Omega, & \left.\dot{\alpha}_{1}, \dot{\alpha}_{2}\right) \\
= & \frac{1}{2}\left[m_{1} v_{1}^{2}+m_{2} v_{2}^{2}+m_{3} v_{3}^{2}+\bar{I}_{1} \Omega_{1}^{2}+\bar{I}_{2} \Omega_{2}^{2}+\lambda_{3} \Omega_{3}^{2}+\frac{1}{\sigma_{1}} J_{1} \Omega_{1}^{2}+\frac{1}{\sigma_{2}} J_{2} \Omega_{2}^{2}\right. \\
& \left.+\frac{\sigma_{1}}{\sigma_{1}-1} J_{1}\left(\Omega_{1}+\dot{\alpha}_{1}-\frac{1}{\sigma_{1}} \Omega_{1}\right)^{2}+\frac{\sigma_{2}}{\sigma_{2}-1} J_{2}\left(\Omega_{2}+\dot{\alpha}_{2}-\frac{1}{\sigma_{2}} \Omega_{2}\right)^{2}\right]
\end{aligned}
$$


where $\sigma_{1}, \sigma_{2}$ are free variables and matching is ensured by Theorem 2.1. The controlled conserved quantities are for $i=1,2$

$$
\tilde{l}_{i}=\frac{\partial l_{\tau, \sigma, \rho}}{\partial \dot{\alpha}_{i}}=J_{i} \Omega_{i}+\rho_{i} J_{i} \dot{\alpha}_{i}
$$

Control law: Using (24), the control law is

$$
\begin{aligned}
& u_{1}=\frac{1}{\sigma_{1}} J_{1} \dot{\Omega}_{1} \\
& u_{2}=\frac{1}{\sigma_{2}} J_{2} \dot{\Omega}_{2}
\end{aligned}
$$

To get the control law with accelerations eliminated we use formula (32). We compute from (29) that the matrix $B$ is diagonal and has diagonal elements $\left(m_{1}, m_{2}, m_{3}, \bar{I}_{1}, \bar{I}_{2}, \lambda_{3}\right)$. We then compute $D$ from (30) to be

$$
\left(\begin{array}{cc}
D^{\alpha_{1} \alpha_{1}} & D^{\alpha_{1} \alpha_{2}} \\
D^{\alpha_{2} \alpha_{1}} & D^{\alpha_{2} \alpha_{2}}
\end{array}\right)=\left(\begin{array}{cc}
\frac{1}{J_{1}}+\frac{1}{\sigma_{1}} \frac{1}{\bar{I}_{1}} & 0 \\
0 & \frac{1}{J_{2}}+\frac{1}{\sigma_{2}} \frac{1}{\bar{I}_{2}}
\end{array}\right)
$$

Using (31), we find the control gains $k_{a}^{\alpha}$. The only non-zero elements of this gain matrix are

$$
\begin{aligned}
& k_{1}=k_{\alpha_{1}}^{\Omega_{1}}=\frac{J_{1}}{\sigma_{1} \bar{I}_{1}+J_{1}} \\
& k_{2}=k_{\alpha_{2}}^{\Omega_{2}}=\frac{J_{2}}{\sigma_{2} \bar{I}_{2}+J_{2}}
\end{aligned}
$$

Using (32), the control law is

$$
\begin{aligned}
& u_{1}=k_{1}\left(\left(\lambda_{2}-\lambda_{3}\right) \Omega_{2} \Omega_{3}+J_{2} \dot{\alpha}_{2} \Omega_{3}+\left(m_{2}-m_{3}\right) v_{2} v_{3}\right) \\
& u_{2}=k_{2}\left(\left(\lambda_{3}-\lambda_{1}\right) \Omega_{3} \Omega_{1}+J_{1} \dot{\alpha}_{1} \Omega_{3}+\left(m_{3}-m_{1}\right) v_{3} v_{1}\right) .
\end{aligned}
$$

Stabilization: The family of relative equilibria that we are interested in stabilizing with the two given rotors corresponds to translation along and rotation about the third principal axis of the vehicle, i.e.

$$
v=\left[\begin{array}{l}
0 \\
0 \\
\bar{v}
\end{array}\right], \quad \Omega=\left[\begin{array}{l}
0 \\
0 \\
\bar{\Omega}
\end{array}\right], \quad \dot{\alpha}=\left[\begin{array}{l}
0 \\
0
\end{array}\right]
$$

where $\bar{v} \neq 0$. For example, if the third principal axis corresponds to the longest physical axis of the vehicle (i.e. longest semi-axis of an ellipsoidal vehicle), then for the uncontrolled vehicle, this family of relative equilibria is unstable (except possibly linearly stable in a region of large values of $\bar{\Omega} / \bar{v}$ as described in Reference [24]). Typically, such a motion is stabilized by propellers and fins. Internal rotors are advantageous since they are not exposed 
to the harsh seawater environment and they can provide stabilization even at low vehicle velocities.

We will consider here the case in which the third principal axis is the longest physical axis of the vehicle and the first principal axis is the shortest. This implies that

$$
m_{3}<m_{2}<m_{1}
$$

Two Casimir functions for this problem are as follows:

$$
\begin{aligned}
& C_{1}=P_{1} \Pi_{1}+P_{2} \Pi_{2}+P_{3} \Pi_{3} \\
& C_{2}=\frac{1}{2}\left(P_{1}^{2}+P_{2}^{2}+P_{3}^{2}\right)
\end{aligned}
$$

Thus, our Lyapunov function for studying stability becomes

$$
E_{\tilde{\Phi}, \Psi}=l_{\tau, \sigma, \rho}+\tilde{\Phi}\left(C_{1}, C_{2}\right)+\Psi\left(\tilde{l}_{1}, \tilde{l}_{2}\right)
$$

To satisfy (45) for Theorem 2.2, the first partial derivatives of $\tilde{\Phi}$ evaluated at the equilibrium should satisfy

$$
\begin{aligned}
& \left.\tilde{\Phi}^{\prime}\right|_{e}=\left.\frac{\partial \tilde{\Phi}}{\partial C_{1}}\right|_{e}=-\frac{\bar{\Omega}}{m_{3} \bar{v}} \\
& \left.\dot{\tilde{\Phi}}\right|_{e}=\left.\frac{\partial \tilde{\Phi}}{\partial C_{2}}\right|_{e}=-\frac{1}{m_{3}}+\frac{\lambda_{3} \bar{\Omega}^{2}}{m_{3}^{2} \bar{v}^{2}}
\end{aligned}
$$

Given this criterion, we need only show that we can make $N_{\alpha \beta}=G_{\alpha \beta}+G_{\alpha \gamma} \tilde{H}^{\gamma \delta} G_{\delta \beta}$ definite. In this case we compute the matrix with elements $G_{\alpha \beta}=g_{\alpha \beta}-g_{a \alpha} \rho^{a b} g_{b \beta}$ to be the diagonal matrix $\operatorname{diag}\left(m_{1}, m_{2}, m_{3}, \bar{I}_{1}+J_{1} / \sigma_{1}, \bar{I}_{2}+J_{2} / \sigma_{2}, \lambda_{3}\right)$. The matrix with elements $\tilde{H}^{\alpha \beta}$ as defined by (49) is computed to be

$$
\tilde{H}=\left(\begin{array}{cccccc}
\left.\dot{\tilde{\Phi}}\right|_{e} & 0 & 0 & \left.\tilde{\Phi}^{\prime}\right|_{e} & 0 & 0 \\
0 & \left.\dot{\tilde{\Phi}}\right|_{e} & 0 & 0 & \left.\tilde{\Phi}^{\prime}\right|_{e} & 0 \\
0 & 0 & \tilde{H}^{33} & 0 & 0 & \tilde{H}^{36} \\
\left.\tilde{\Phi}^{\prime}\right|_{e} & 0 & 0 & 0 & 0 & 0 \\
0 & \left.\tilde{\Phi}^{\prime}\right|_{e} & 0 & 0 & 0 & 0 \\
0 & 0 & \tilde{H}^{36} & 0 & 0 & \tilde{H}^{66}
\end{array}\right)
$$

where

$$
\begin{aligned}
\tilde{H}^{33} & =\left.\dot{\tilde{\Phi}}\right|_{e}+\left.\tilde{\Phi}^{\prime \prime}\right|_{e} \lambda_{3}^{2} \bar{\Omega}^{2}+\left.2 \dot{\tilde{\Phi}}^{\prime}\right|_{e} \lambda_{3} \bar{\Omega} m_{3} \bar{v}+\left.\ddot{\widetilde{\Phi}}\right|_{e} m_{3}^{2} \bar{v}^{2} \\
\tilde{H}^{36} & =\left.\tilde{\Phi}^{\prime}\right|_{e}+\left.\tilde{\Phi}^{\prime \prime}\right|_{e} \lambda_{3} \bar{\Omega} m_{3} \bar{v}+\left.\dot{\tilde{\Phi}}^{\prime}\right|_{e} m_{3}^{2} \bar{v}^{2} \\
\tilde{H}^{66} & =\left.\tilde{\Phi}^{\prime \prime}\right|_{e} m_{3}^{2} \bar{v}^{2}
\end{aligned}
$$


Using this, we compute the matrix with elements $N_{\alpha \beta}$ to be

$$
N=\left(\begin{array}{cccccc}
m_{1}+\left.m_{1}^{2} \dot{\tilde{\Phi}}\right|_{e} & 0 & 0 & \left.m_{1} \Delta_{1} \tilde{\Phi}^{\prime}\right|_{e} & 0 & 0 \\
0 & m_{2}+\left.m_{2}^{2} \dot{\tilde{\Phi}}\right|_{e} & 0 & 0 & \left.m_{2} \Delta_{2} \tilde{\Phi}^{\prime}\right|_{e} & 0 \\
0 & 0 & m_{3}+m_{3}^{2} \tilde{H}^{33} & 0 & 0 & m_{3} \lambda_{3} \tilde{H}^{36} \\
\left.m_{1} \Delta_{1} \tilde{\Phi}^{\prime}\right|_{e} & 0 & 0 & \Delta_{1} & 0 & 0 \\
0 & \left.m_{2} \Delta_{2} \tilde{\Phi}^{\prime}\right|_{e} & 0 & 0 & \Delta_{2} & 0 \\
0 & 0 & m_{3} \lambda_{3} \tilde{H}^{36} & 0 & 0 & \lambda_{3}+\lambda_{3}^{2} \tilde{H}^{66}
\end{array}\right)
$$

where

$$
\Delta_{i}=\bar{I}_{i}+\frac{J_{i}}{\sigma_{i}}=\frac{\bar{I}_{i}}{1-k_{i}}
$$

The first three diagonal elements of $N$ are

$$
N_{11}=m_{1}^{2}\left(\frac{1}{m_{1}}-\frac{1}{m_{3}}\right)<0, \quad N_{22}=m_{2}^{2}\left(\frac{1}{m_{2}}-\frac{1}{m_{3}}\right)<0, \quad N_{33}=\left.\ddot{\tilde{\Phi}}\right|_{e} m_{3}^{4} \bar{v}^{2}<0
$$

where we have used the fact that $m_{3}<m_{2}<m_{1}$ and have chosen $\left.\ddot{\widetilde{\Phi}}\right|_{e}<0$. Since these first three diagonal element are negative, we seek to make $N_{\alpha \beta}$ negative definite.

At this point we specialize to the equilibrium in which $\bar{\Omega}=0$. This is a practical choice as it corresponds to the vehicle translating along its long axis but not spinning. For this equilibrium, $N_{\alpha \beta}$ is negative definite if we take

$$
\left.\dot{\tilde{\Phi}}^{\prime}\right|_{e}=0,\left.\quad \tilde{\Phi}^{\prime \prime}\right|_{e}<-\frac{1}{\lambda_{3} m_{3}^{2} \bar{v}^{2}}, \quad \Delta_{1}<0, \quad \Delta_{2}<0 .
$$

The conditions on $\Delta_{1}$ and $\Delta_{2}$ hold if and only if $k_{1}>1$ and $k_{2}>1$. Therefore, by Theorem 2.2 we have proved.

\section{Proposition 3.2}

For $k_{1}>1$ and $k_{2}>1$, the equilibrium $(0,0, \bar{v}, 0,0,0,0,0)$, for $\bar{v} \neq 0$, is nonlinearly stable for the feedback controlled system where $u$ is given by (81).

Asymptotic stability and dissipative controls for the underwater vehicle with rotors are discussed in detail in Reference [19] and in the presence of viscous fluid drag in Reference [25].

\section{CONCLUSIONS AND FUTURE DIRECTIONS}

We expect that the techniques in this paper can be combined with those of Leonard [26] and Bloch et al. [27, 7] who introduced and developed symmetry-breaking potentials for purposes of stabilization as well as tracking. In the context of the satellite with rotors, for example, symmetry breaking may allow for attitude control as well as spin stabilization. Jalnapurkar and Marsden $[27,28]$, which put the potential shaping work of van der Shaft into the context of mechanical systems with symmetry, may also be useful in this regard. 


\section{ACKNOWLEDGEMENTS}

We thank John Baillieul, Francesco Bullo, Joel Burdick, Dong Eui Chang, P.S. Krishnaprasad, Richard Murray, Tudor Ratiu, Gloria Sánchez de Alvarez and Craig Woolsey for helpful comments. AMB would like to acknowledge partial research support by the National Science Foundation grant DMS-9803181 and AFOSR grant F49620-96-1-0100, NEL acknowledges partial support from the National Science Foundation under grant BES-9502477 and by the Office of Naval Research under grant N00014-98-1-0649 and JEM acknowledges partial support from the California Institute of Technology and AFOSR Grant F49620-95-1-0419.

\section{REFERENCES}

1. Bloch AM, Leonard NE, Marsden JE. Stabilization of mechanical systems using controlled Lagrangians, Proceedings of the IEEE Conference on Decision and Control 1997; 36:2356-2361.

2. Bloch AM, Leonard NE, Marsden JE. Matching and stabilization by the method of controlled Lagrangians. Procedings of the IEEE Conference on Decision and Control 1998; 37:1446-1451.

3. Bloch AM, Leonard NE, Marsden JE. Stabilization of the pendulum on a rotor arm by the method of controlled Lagrangians, Proceedings of the International Conference on Robotics and Automation 1999; 500-505.

4. Bloch AM, Leonard NE, Marsden JE. Potential shaping and the method of controlled Lagrangians. Proceedings of the IEEE Conference on Decision and Control 1999; 38:1653-1657.

5. Bloch AM, Leonard NE, Marsden JE. Controlled Lagrangians and the stabilization of mechanical systems I: The first matching theorem. IEEE Transactions on Automatic Control 2000; 45:2253-2270.

6. Bloch AM, Chang DE, Leonard NE, Marsden JE. Controlled Lagrangians and the stabilization of mechanical systems II: potential shaping and tracking, 2000, IEEE Transactions on Automatic Control, to appear.

7. Bloch AM, Chang DE, Leonard NE, Marsden JE, Woolsey CA. Asymptotic stabilization of Euler-Poincaré mechanical systems. Proceedings of the IFAC Workshop on Lagrangian and Hamiltonian Methods in Nonlinear Control. Elsevier: Amsterdam; 2000; 56-61.

8. Krishnaprasad PS. Lie-Poisson structures, dual-spin spacecraft and asymptotic stability. Nonlinear Analysis Theory Methods and Applications 1985; 9:1011-1035.

9. van der Schaft AJ. Stabilization of Hamiltonian systems. Nonlinear Analysis, Theory, Methods and Applications 1986; 10:1021-1035.

10. Åström KJ, Furuta K. Swinging up a pendulum by energy control. IF AC, San Francisco, vol. 13, 1996.

11. Wang LS, Krishnaprasad PS. Gyroscopic control and stabilization. Journal of Nonlinear Science 1992; 2:367-415

12. Koditschek DE. The application of total energy as a Lyapunov function for mechanical control systems. In Dynamics and Control of Multibody Systems (Brunswick, ME, 1988). Contemporary Mathematics vol. 97, American Mathematical Society: Providence, RI, 1989; 131-157.

13. Koditschek, DE, Rimon E. Robot navigation functions on manifolds with boundary. Advances in Applied Mathematics 1990; 11:412-442.

14. Baillieul, J. Stable average motions of mechanical systems subject to periodic forcing. Fields Institute Communications 1993; 1:1-23.

15. Auckly D, Kapitanski L, White W. Control of nonlinear underactuated systems. Communication in Pure and Applied Mathematics 1998; 53:354-369.

16. Hamberg J. General matching conditions in the theory of controlled Lagrangians. Proceedings of the IEEE Conference on Decision and Control 1999; 38:2519-2523.

17. Marsden JE. Lectures on Mechanics. London Mathematical Society Lecture Note Series, 174. Cambridge University Press: Cambridge, 1992.

18. Marsden JE, Ratiu TS. Symmetry and Mechanics. Texts in Applied Mathematics, (2nd edn), vol. 17. Springer: Berlin, 1999.

19. Woolsey CA, Leonard NE. Underwater vehicle stabilization by internal rotors. Proceedings American Control Conference 1999; 3417-3421.

20. Bloch AM, Krishnaprasad PS, Marsden JE, Sánchez de Alvarez G. Stabilization of rigid body dynamics by internal and external torques. Automatica 1992; 28:745-756.

21. Leonard, NE. Stability of a bottom-heavy underwater vehicle Automatica 1997; 33:331-346.

22. Leonard NE, Marsden JE. Stability and drift of underwater vehicle dynamics: Mechanical systems with rigid motion symmetry. Physica D 1997; 105:130-162.

23. Leonard NE, Woolsey CA. Internal actuation for intelligent underwater vehicle control, Proceedings of the 10th Yale Workshop on Adaptive and Learning Systems 1998; 295-300.

Copyright (C) 2001 John Wiley \& Sons, Ltd.

Int. J. Robust Nonlinear Control 2001; 11:191-214 
24. Holmes P, Jenkins J, Leonard NE. Dynamics of the Kirchhoff equations I: coincident centers of gravity and buoyancy. Physica D 1998; 118:311-342.

25. Woolsey CA, Leonard NE. Global asymptotic stabilization of an underwater vehicle using internal rotors. Proceedings of the IEEE Conference on Decision and Control 1999; 38:2527-2532.

26. Leonard NE. Stabilization of underwater vehicle dynamics with symmetry-breaking potentials, Systems and Control Letters 1997; 32:35-42.

27. Jalnapurkar SM, Marsden JE Stabilization of relative equilibria II, Regular and Chaotic Dynamics 1999; 3:161-179.

28. Jalnapurkar SM, Marsden JE. Stabilization of relative equilibria I, IEEE Trans. Automatic Control 2000, 45: 1483-1491.

29. Bloch AM, Krishnaprasad PS, Marsden JE, Ratiu TS. Dissipation induced instabilities, Annales de l'Institut Henri Poincaré, Analyse Non Linéaire 1993; 11:37-90.

30. Bloch AM, Marsden JE, Sánchez G. Stabilization of relative equilibria of mechanical systems with symmetry. In Current and Future Directions in Applied Mathematics, Alber M, Hu B, Rosenthal J. (eds). Birkhäuser: Basel, 43-64.

31. Koon WS, Marsden JE. The Hamiltonian and Lagrangian approaches to the dynamics of nonholonomic systems. Reportion Mathamatical Physics 1997; 40:21-62.

32. Marsden JE, Scheurle J. The reduced Euler-Lagrange equations. Fields Institute Communications 1993; 1:139-164. 